\title{
ANALYSIS OF F AND G SUBDWARFS. I. THE LOCATION OF SUBDWARFS IN THE THEORETICAL H-R DIAGRAM
}

\author{
S. E. Strom And J. G. Cohen \\ Smithsonian Astrophysical Observatory and Harvard College Observatory, \\ Cambridge, Massachusetts \\ AND \\ K. M. STROM \\ Smithsonian Astrophysical Observatory, Cambridge, Massachusetts \\ Received August 10, 1966
}

\begin{abstract}
Spectrum scans and model stellar atmospheres have been used to locate in the $\left(L / L \odot, T_{\text {eff }}\right)$ plane $F$ and $G$ subdwarfs having known parallax A comparison is made between their position on the $H-R$ diagram and the evolutionary tracks for Population II models of low- and high-helium content recently computed by Faulkner and Iben. The lower helium-content models are found to give somewhat more plausible ages than the high-helium models. Nevertheless, for both the low-and high-helium-content models, we find several subdwarfs with ages apparently greater than $10 \times 10^{9}$ years.
\end{abstract}

\section{INTRODUCTION}

The spectra, kinematical properties, and spatial distribution of subdwarfs indicate that these Population II objects may be among the oldest in our Galaxy. They are consequently of great significance in discussing many cosmogonic problems. However, the exact location of subdwarfs in the theoretical H-R diagram is essential to a discussion of their evolutionary history. Before their weak-line spectra were understood in terms of real abundance deficiencies, it was thought that the subdwarfs formed a distinct group that lay below the Population I main sequence by up to 3 magnitudes. The weak lines led to a choice of too early a spectral type, and, as a consequence, the subdwarfs were located systematically to the left in the H-R diagram. The work of Chamberlain and Aller (1951), the Schwarzschilds (1950), and the Burbidges (1956) was essential in demonstrating quantitatively that element abundances in subdwarfs range from nearly solar values to factors of 100 and more below solar values. But, owing to the weakness of the metallic lines, it was still difficult to assign accurate spectral types and colors to subdwarfs and thus difficult to place these stars on the $\left(M_{\mathrm{bol}}, \log T_{\text {eff }}\right)$ diagram.

Schwarzschild, Searle, and Howard (1955) were first to suggest that a difference in line blanketing between the subdwarfs and the more metal-rich Population I stars could account for the anomalies in the observed colors. Sandage and Eggen (1959) then demonstrated that, within the observational errors, proper blanketing corrections place the subdwarfs on the Population I $\left(M_{v}, B-V\right)$ diagram. Eggen and Sandage later argued that the subdwarfs coincide with the Population I main sequence in the $\left(M_{\text {bol }}, \log T_{\text {eff }}\right)$ plane as well. Melbourne (1960) combined the results of narrow-band-pass spectrum scans and high-dispersion spectra to obtain line-free monochromatic magnitudes for a small sample of Population I stars and subdwarfs. He then compared these measures with a rather crude grid of model atmospheres and obtained values for the effective temperature, which he then corrected by a factor depending on the total flux "removed" by the lines. His results supported the conclusions of Eggen and Sandage (1962) regarding the location of subdwarfs on the $\left(M_{\text {bol }}, T_{\text {eff }}\right)$ plane.

In principle, Melbourne's method provides the most accurate means available for choosing values of $T_{\text {eff. }}$. Since Melbourne's work appeared, many more spectrum scans of subdwarfs have become available. In addition, more modern model-atmosphere

1038 
computations have attained a much greater degree of sophistication. In view of these recent observational and theoretical advances, we wish to discuss in this paper the location of subdwarfs in the theoretical H-R diagram. Furthermore, by using abundance analyses based on model atmospheres, we may hope to improve on the previous abundance determinations that were based on the classical curve-of-growth methods and thus to discuss more definitively some problems of importance in understanding stellar element synthesis. The question of subdwarf abundances is examined in "Paper II" (Strom and Cohen 1966).

\section{THE POSITION OF THE SUBDWARFS ON THE THEORETICAL H-R DIAGRAM}

\section{a) The Observational Data}

We have chosen to determine values of $T_{\text {eff }}$ for all the stars discussed by Sandage and Eggen (1959) for which spectrum scans were available. In all cases, photoelectric $V$ magnitudes have been measured, the parallax $\pi$ exceeds 0.05 , and each parallax represents a weighted average (Eggen 1955) of observations from more than one observatory.

TABLE 1

OBSERVATIONAL DATA

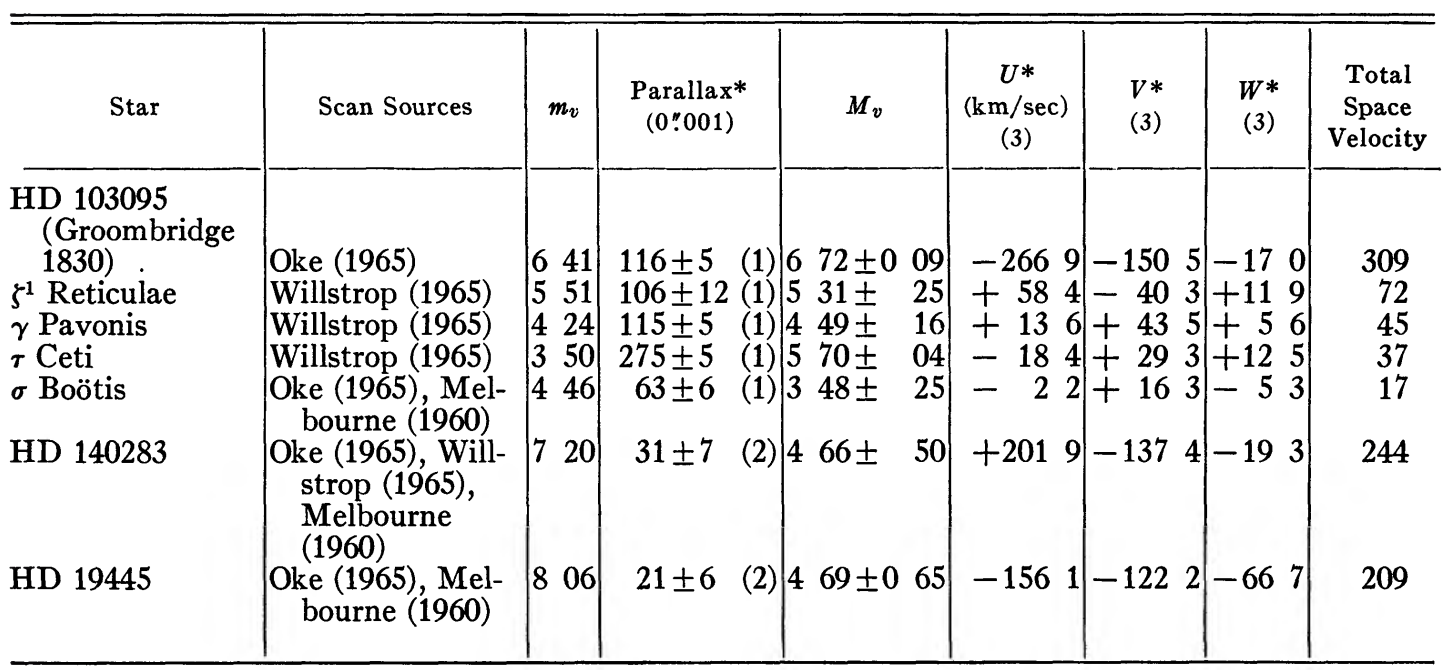

* Sources: (1) Sandage and Eggen (1959); (2) Aller and Greenstein (1960); (3) Eggen (1962).

We have, in addition, included in our study the two extreme subdwarfs HD 140283 and HD 19445 for which $\pi=0 " .031 \pm 0.007$ and $\pi=0.021 \pm 0.006$, respectively, since they have been the object of intensive spectrophotometric investigation. It would be of great importance to have available more accurate parallax determinations for these stars.

The spectrum scans used in this investigation are those of Willstrop (1965), Oke (1965), and Melbourne (1960).

The observed quantities and their source are summarized in Table 1.

Comparison between the results obtained for these subdwarfs and for other stars common to the lists of these authors is extremely encouraging insofar as relative fluxes for wavelengths longer than $4000 \AA$ are concerned. For relative fluxes shortward of the Balmer discontinuity, when available, Oke's (1965) suggested calibration is adopted. However, as Oke indicates, the Balmer discontinuities resulting from this calibration may be too small by 0.05 or 0.06 mag. This must be considered when we discuss in Paper II the choice of surface gravity as dictated by the Balmer discontinuity. 


\section{b) The Model Atmospheres}

The stellar-atmosphere program developed by Strom and Avrett (1965) has been used in computing a grid of LTE stellar models in the effective temperature range of $5000^{\circ}<T_{\text {eff }}<7000^{\circ} \mathrm{K}$ and for surface gravities $\log g=3.0$ to 5.00. The metal abundances were chosen to be 1/100 times the GMA (Goldberg, Müller, and Aller 1960) solar values. The slope of the Paschen continuum is extremely sensitive to temperature as shown in Figure 1, where the monochromatic magnitudes per unit frequency relative to $\lambda 5560 \AA$ are plotted for the indicated values of $T_{\text {eff. }}$. The flux in the Paschen continuum for a model of given $T_{\text {eff }}$ is insensitive to changes in $\log g$ or metal abundance. The agreement with the LTE atmosphere calculations of Swihart and Fischel (1961) is to within a few per cent for the temperature distribution and the emergent fluxes.

It is of interest to note that, even with 0.01 the solar abundance, $\mathrm{Si}$ and $\mathrm{Mg}$ still provide several per cent of the total opacity in the vicinity of the Balmer discontinuity and

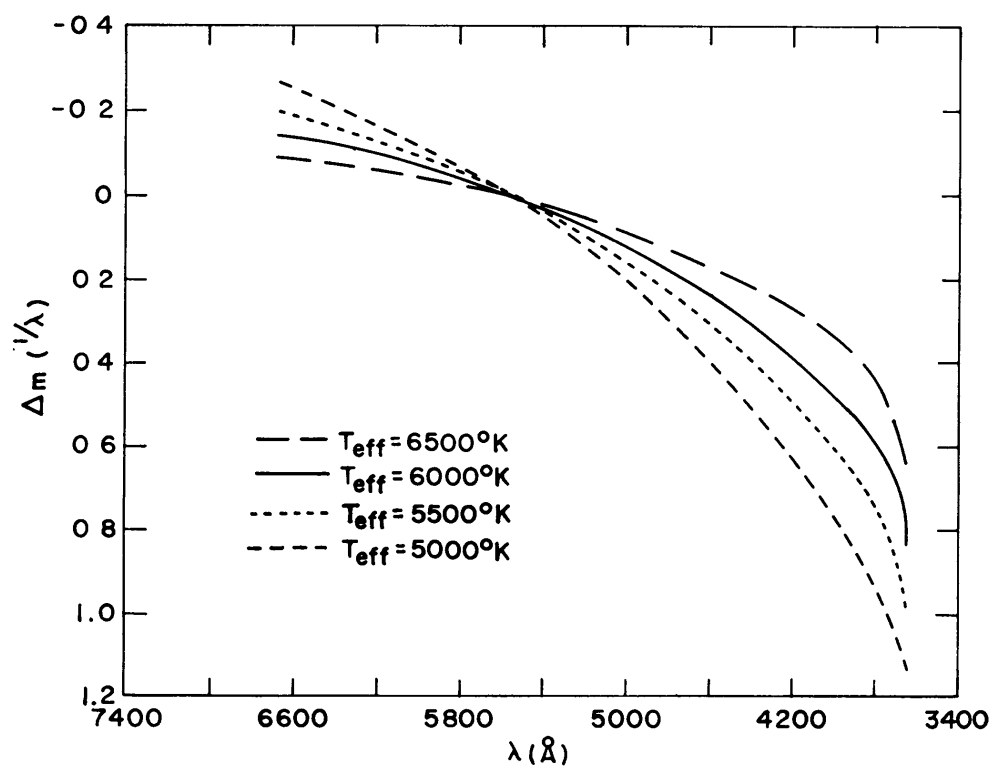

Fig. 1.-Comparison of the predicted monochromatic magnitudes from four LTE unblanketed model stellar atmospheres.

significantly more in the ultraviolet, according to Gingerich and Rich's (1967) tabulation of these metal-absorption coefficients.

The effect of line blanketing has been omitted in these computations. By using the table of atomic parameters for 31000 line transitions of astrophysical interest described by Strom and Kurucz (1966), we have computed for 10- $\AA$ regions centered at 3400, 4190, and $4590 \AA$ the line-to-continuum opacity ratio as a function of optical depth for a $\left(5500^{\circ} \mathrm{K}, \log g=4,0.01\right.$ GMA metals) model. We find that the amount of energy "removed" by the lines in these regions is at most a few per cent and that indeed these are "windows" in the sense that we are observing the true continuum. This is also the case for 0.1 GMA abundances. In both cases, the blocking of radiation by lines for $\lambda>$ $5000 \AA$ is negligible. At 0.01 GMA the effect of line blanketing in all observable regions of the spectrum amounts to about 1 per cent as Melbourne (1960) has shown for HD 140283. However, for 0.1 GMA, the fraction of energy blocked by the lines is about 10 per cent. Thus, for metal abundance greater than 0.01 GMA, the value of effective temperature $T^{M}$ eff deduced by fitting a model atmopshere to the fluxes observed in the windows will be too high. 
As a zero-order estimate of the effect of line blanketing on the deduced $T_{\text {eff }}$, we follow the procedure summarized by Melbourne (1960). If $\Delta F$ is the fraction of energy removed by the lines as measured on high-dispersion spectra, then the ratio of the true effective temperature, $T^{*}{ }_{\text {eff }}$, to $T^{M}{ }_{\text {eff }}$ is

$$
T^{*}{ }_{\text {eff }} / T^{M}{ }_{\text {eff }}=(1-\Delta F)^{1 / 4} .
$$

In Table 2 we summarize the values of this ratio and its logarithm for typical $\Delta F$ values. For subdwarfs in the $T_{\text {eff }}$ range from $5000^{\circ}$ to $7000^{\circ} \mathrm{K}$, we therefore expect the maximum adjustment in the $T^{M}$ eff values to amount to 0.02 in the log.

In a recent paper Kalkofen and Strom (1966) have computed and discussed a stellar model in which the LTE assumption has been relaxed for $\mathrm{H}^{\text {and }} \mathrm{H}^{-}$. We have extended their methods of computation to estimate the maximum effect of departures from LTE on the emergent continuum fluxes for a typical subdwarf atmosphere. In Figure 2 we plot the emergent monochromatic magnitudes for LTE and non-LTE $\left(5500^{\circ}, 3.7,0.01\right)$ models and an LTE $\left(6000^{\circ}, 3.7,0.01\right)$ model. The essential feature of this plot is that the non-LTE $\left(5500^{\circ}, 3.7,0.01\right)$ model lies between the LTE $\left(6000^{\circ}, 3.7,0.01\right)$ and $\left(5500^{\circ}\right.$, $3.7,0.01$ ) models. Thus, we can state qualitatively that the use of LTE models leads to a choice of too high a value of $T_{\text {eff }}$.

TABLE 2

APPROXIMATE LINE-BLANKETING CORRECTIONS

\begin{tabular}{c|c|c}
\hline \multicolumn{1}{c|}{$\Delta F$} & $T^{*}{ }_{\text {eff }} / T^{M}$ eff & $\log T^{*}{ }_{\text {eff }} / T^{M}$ eff \\
\hline 020 & 0946 & -0024 \\
15 & 960 & -018 \\
0.10 & 974 & -011 \\
005 & 0985 & -0006 \\
\hline
\end{tabular}

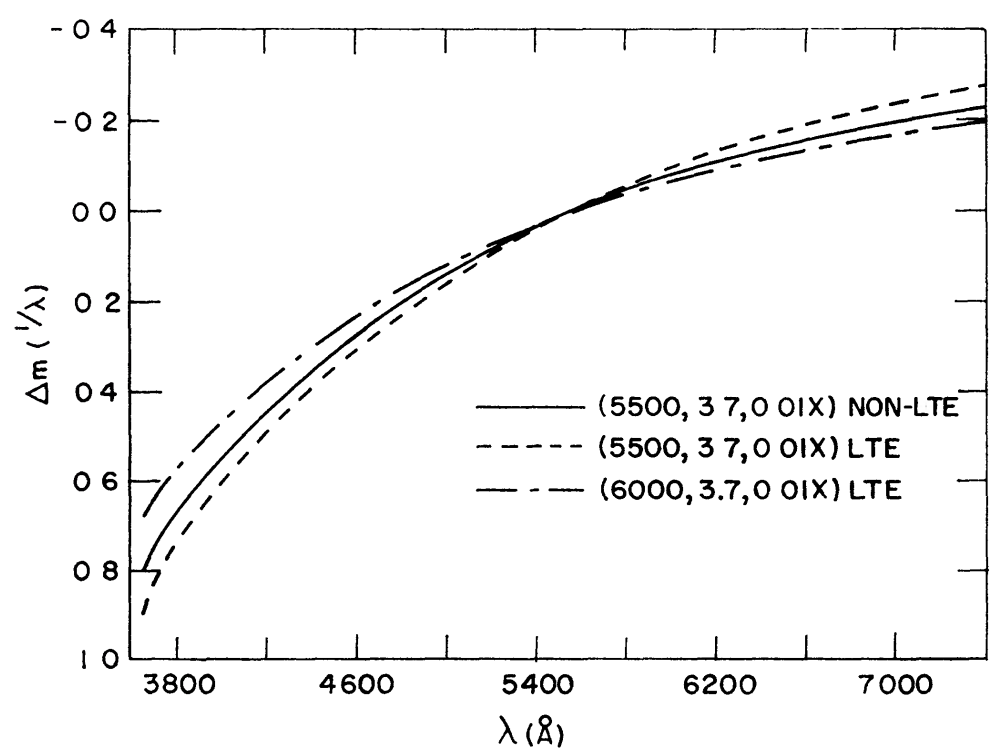

FIG. 2 -Comparison of the predicted monochromatic magnitudes from 2 LTE atmospheres and a non-LTE atmosphere. 
A more definitive statement regarding the magnitude of the effects of departures from LTE depends on knowledge of the rate for the associative detachment reaction $\mathrm{H}^{-}+\mathrm{H} \rightleftharpoons \mathrm{H}_{2}+e$. Unfortunately, estimates of this rate differ widely, and no reliable experimental determinations are available. To obtain a maximum estimate of the deviations from LTE, we have assumed in our calculations that no $\mathrm{H}^{-}$is destroyed by this reaction, and only radiative destruction of $\mathrm{H}^{-}$is important. We can conclude from our model that it is possible that we are overestimating $T_{\text {eff }}$ by as much as 0.02 in the $\log$ by ignoring departures from LTE.

It is important to note that by including line blanketing or departures from LTE the tendency in both cases is to lower the estimates of $T_{\text {eff }}$ obtained from the LTE unblanketed models.

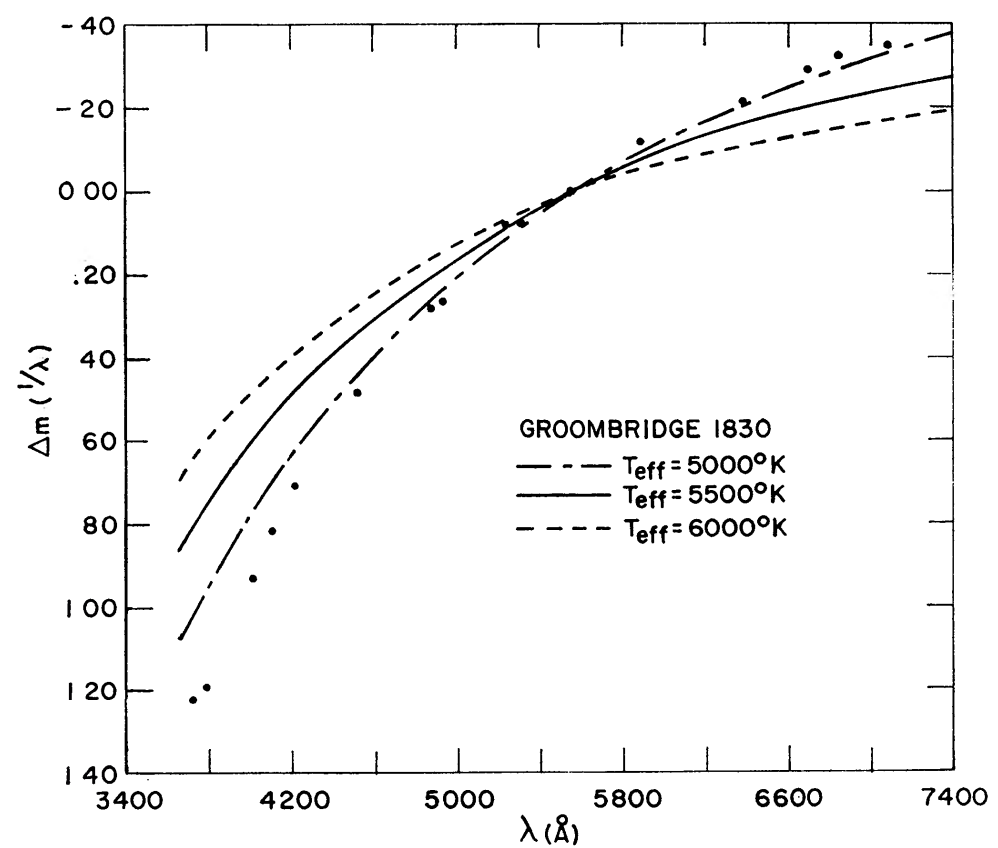

FIG. 3.-Comparison between the monochromatic magnitudes observed by Oke (1965) for Groombridge 1830 and those predicted from LTE unblanketed model stellar atmospheres.

\section{c) The Effective Temperature Determinations}

In Figures 3-9 we compare the observed monochromatic magnitude per unit frequency relative to $5560 \AA$ with those computed from the LTE unblanketed stellar models described in the previous section. The values of $T_{\text {eff }}$ deduced are summarized in Table 3. In all cases, we must recall that these $T_{\text {eff }}$ determinations represent upper limits and may be too high by as much as 0.02 or 0.03 in the log.

In the cases of $\mathrm{HD} 19445$ and $\mathrm{HD} 140283, \mathrm{H} \gamma$ profiles are available (Aller and Greenstein 1960) (Fig. 10). For $T_{\text {eff }}<8000^{\circ} \mathrm{K}$, the $\mathrm{H} \gamma$ profile or equivalent width is a sensitive indicator of $T_{\text {eff }}$ and, moreover, is very insensitive to changes of electron pressure in the stellar atmospheres (Searle and Oke 1962). We have determined, for these stars, values of $T_{\text {eff }}$ from the $\mathrm{H} \gamma$ profiles, and the results are compared with the scanner $T_{\text {eff }}$ values in Table 3. In both cases, the values of $T_{\text {eff }}$ deduced from $\mathrm{H} \gamma$ are about $200^{\circ} \mathrm{K}$ higher than the corresponding scanner $T_{\text {eff }}$. Danziger $(1966 a)$ has reported similar experiences with a larger sample of stars in this $T_{\text {eff }}$ range. One possible explanation may lie in the fact that at $\tau \sim 0.5$, where the wings of $\mathrm{H} \gamma$ are formed, the non-LTE atmosphere pre- 


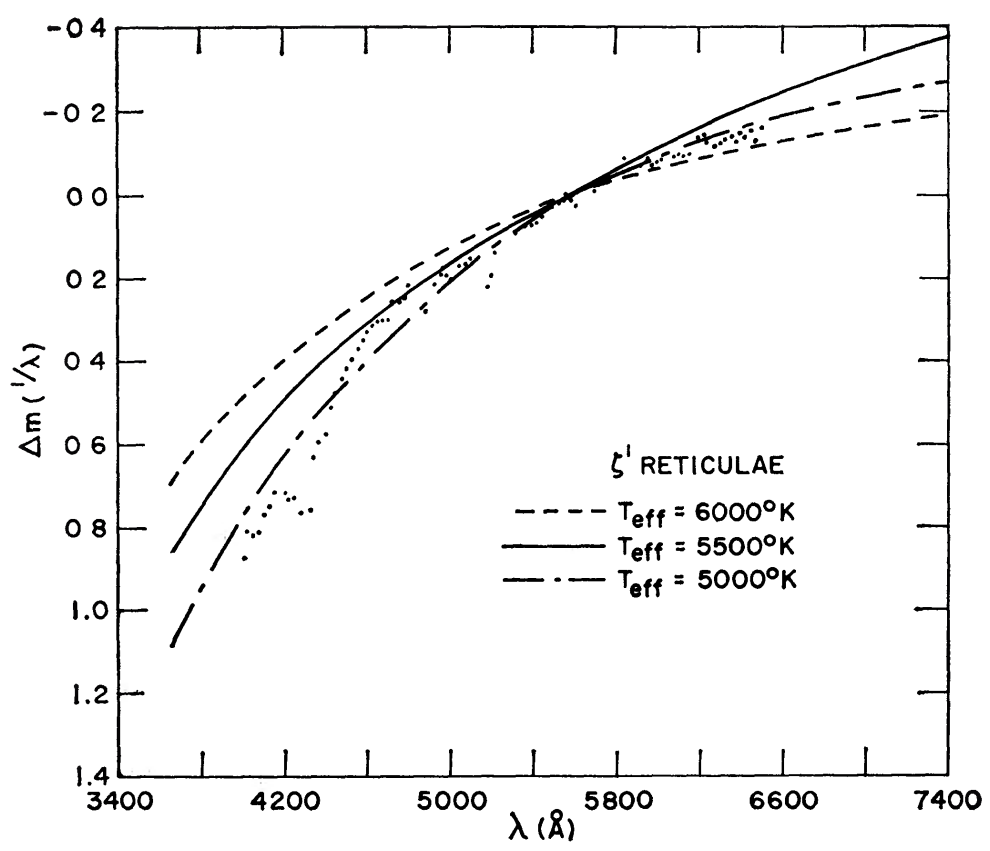

FIG. 4. - Comparison between the monochromatic magnitudes observed by Willstrop (1965) for $\zeta^{1}$ Ret and those predicted from LTE unblanketed model stellar atmospheres.

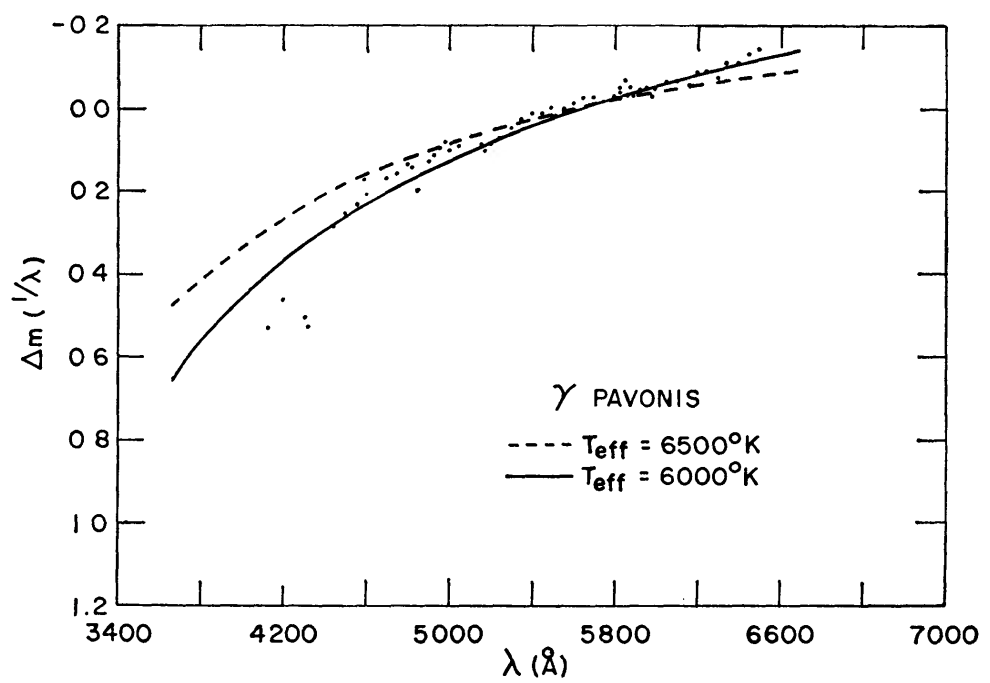

FIG. 5.-Comparison between the monochromatic magnitudes observed by Willstrop (1965) for $\gamma$ Pav and those predicted from LTE unblanketed model stellar atmospheres.

TABLE 3

DETERMINATIONS OF $T_{\text {eff }}$

\begin{tabular}{|c|c|c|c|c|}
\hline Star & $\begin{array}{c}T_{\text {eff }}(\text { scan) } \\
\left({ }^{\circ} \mathrm{K}\right)\end{array}$ & $\begin{array}{c}T_{\text {eff }}(\mathrm{H} \gamma) \\
\left({ }^{\circ} \mathrm{K}\right)\end{array}$ & $\begin{array}{c}T_{\text {eff (ion) }} \\
\left({ }^{\circ} \mathrm{K}\right)\end{array}$ & B C \\
\hline $\begin{array}{l}\text { Groombridge } 1830 \\
\zeta^{1} \text { Reticulae } \\
\gamma \text { Pavonis . } \\
\tau \text { Ceti ... } \\
\sigma \text { Boötis .... } \\
\text { HD 140283 } \\
\text { HD 19445. . . . . }\end{array}$ & $\begin{array}{l}5000 \\
5600 \\
6100 \\
5000 \\
6700 \\
5500 \\
\mathbf{5 7 0 0}\end{array}$ & $\begin{array}{l}5700 \\
5800\end{array}$ & $\begin{array}{l}5750 \\
5900\end{array}$ & $\begin{array}{l}-040 \\
-\quad 30 \\
=\quad 25 \\
=\quad 40 \\
=.20 \\
-\quad .30 \\
-0 \quad 30\end{array}$ \\
\hline
\end{tabular}

1043 


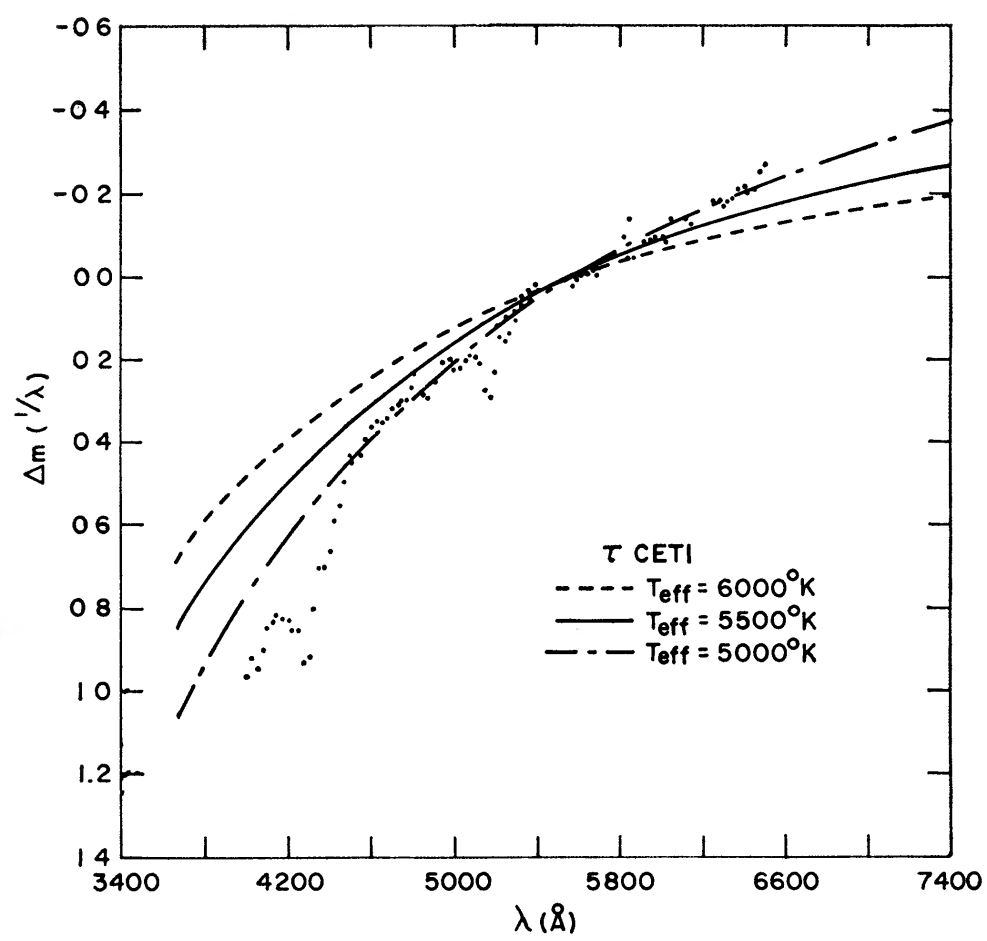

FIG. 6.-Comparison between the monochromatic magnitudes observed by Willstrop (1965) for $\tau$ Ceti and those predicted from LTE unblanketed model stellar atmospheres.

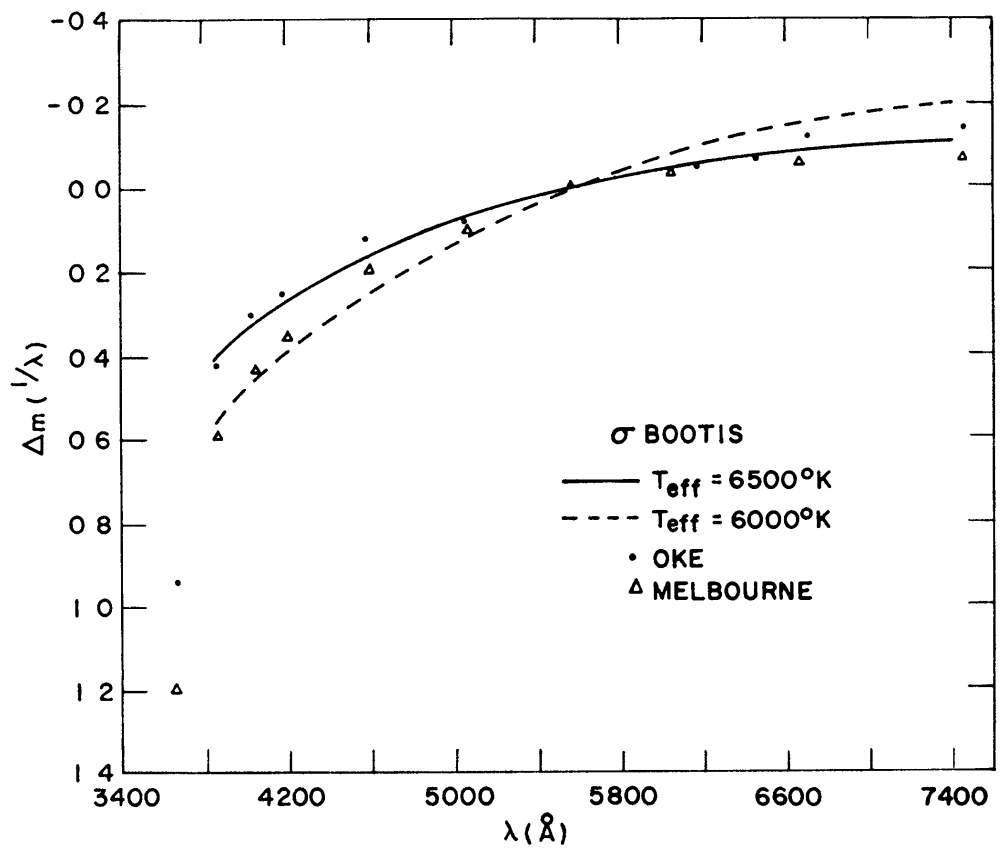

Fig. 7.-Comparison between the monochromatic magnitudes observed by Oke (1965) and Melbourne (1960) for $\sigma$ Boötis and those predicted from LTE unblanketed model stellar atmospheres.

1044 


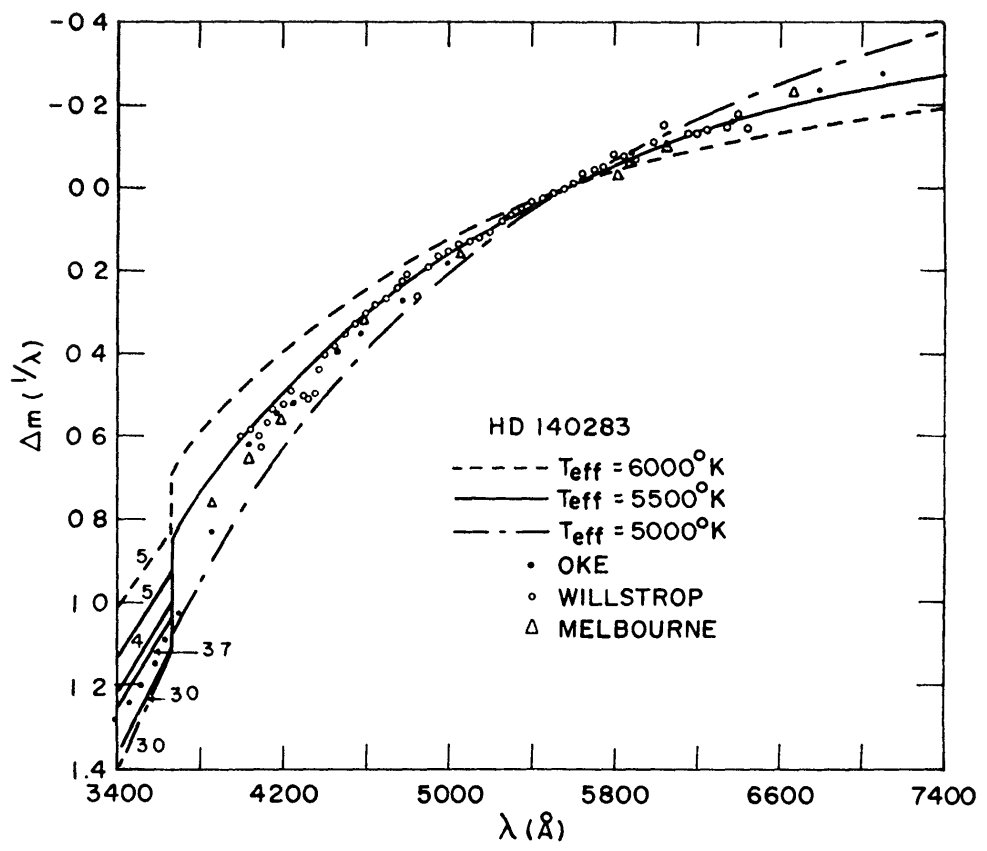

Fig. 8 -Comparison between the monochromatic magnitudes observed by Oke (1965), Willstrop (1965), and Melbourne (1960) for HD 140283 and those predicted from LTE unblanketed model stellar atmospheres. The value of $\log g$ for each model is indicated near the Balmer discontinuity.

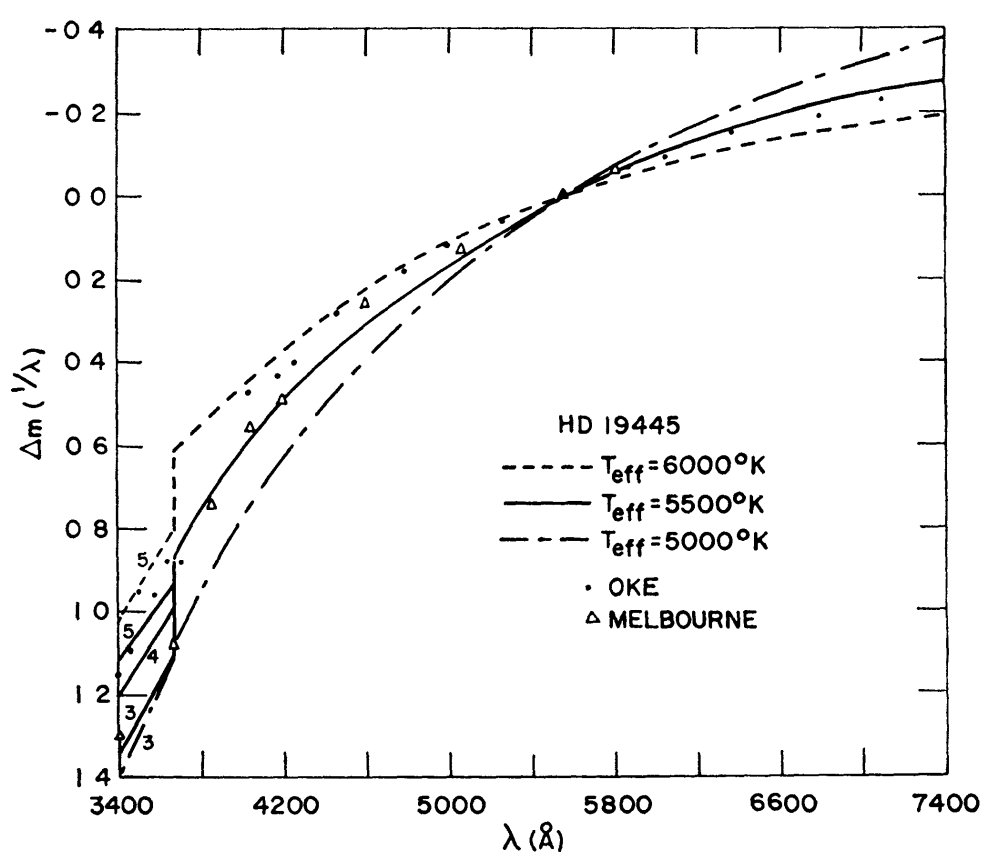

FIG. 9.-Comparison between the monochromatic magnitudes observed by Oke (1965) and Melbourne (1960) for HD 19445 and those predicted from LTE unblanketed model stellar atmospheres. The value of $\log g$ for each model is indicated near the Balmer discontinuity.

1045 
dicts a higher value of temperature than the corresponding LTE atmosphere of the same $T_{\text {eff. }}$ Quantitative evaluations of this effect are under way.

Also indicated in this table are estimates for HD 19445 and HD 140283 of the temperatures giving the best match in the element abundances determined from neutral and singly ionized atoms. A more complete discussion of these estimates appears in Paper II.

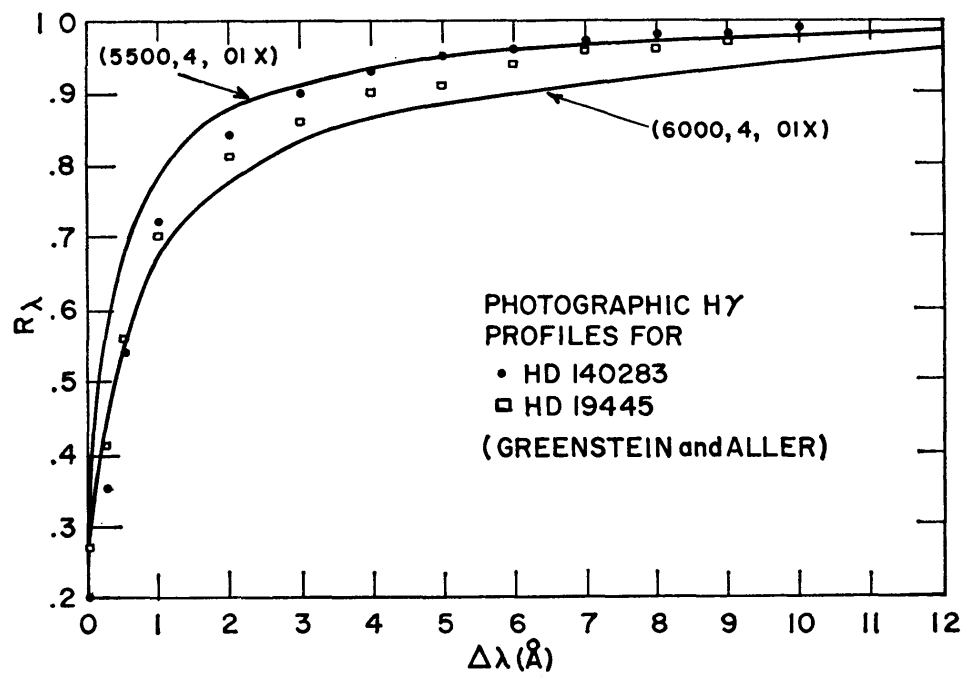

FIG. 10.-Observed $\mathrm{H}_{\gamma}$ profiles for HD 19445 and HD 140283 compared with theoretical $\mathrm{H} \gamma$ profiles calculated from stellar atmospheres having the indicated parameters

TABLE 4

FinAL DETERMINATIONS OF LOG $T_{\text {eff }}$ FOR INDIVIDUAL STARS

Star $\quad \log T_{\text {eff }}$

Groombridge $1830 \quad \ldots \ldots \ldots, 3.70 \pm 0.015$

$\zeta^{1}$ Reticulae............ $375 \pm .015$

$\gamma$ Pavonis $\ldots \ldots \ldots \ldots . . .6 .3 .785 \pm .015$

$\tau$ Ceti.................. 3.70 \pm .015

$\sigma$ Boötis . .......... $3825 \pm .015$

HD $140283 \ldots \ldots . \quad \ldots . .3 .74 \pm .01$

HD $19445 \quad \ldots \ldots . \quad \ldots \quad 3.75 \pm 0.01$

d) Comparison of Evolutionary Tracks and Subdwarf

Positions on the $\left(\log L / L \odot, \log T_{\text {eff }}\right)$ Plane

In order to determine the location of an individual star on an evolutionary track in the $\mathrm{H}-\mathrm{R}$ diagram, we must know the stellar luminosity, effective temperature, and chemical composition.

The observational data summarized in $\S \mathrm{II} a$ allow us to compute absolute visual magnitudes for each of the subdwarfs. To convert to luminosities relative to the solar luminosity, we apply the bolometric corrections listed in Table 3, which follow from emergent fluxes calculated from the stellar atmospheres. The bolometric correction for the Sun is assumed to be -0.11 mag. Owing to line-blanketing effects and errors in the choice of $T_{\text {eff }}$, an individual bolometric correction may be in error by as much as 0.20 mag. However, in most cases, the parallax errors have a greater effect on the uncertainty in the luminosity.

The bases for choosing a value for $T_{\text {eff }}$ are discussed in the previous section, and the individual determinations appear in Table 4. 
From the detailed model-atmosphere analyses performed by us in Paper II for HD 140283 and HD 19445, we conclude that the metal content of these stars is approximately 0.01 times the solar values. Danziger $(1966 b)$ has recently published abundances determined from classical curve-of-growth techniques for $\zeta^{1}$ Ret and $\gamma$ Pav, which indicate that these stars are deficient in metals by factors of 3 and 10, respectively. In another paper, he obtains for $\sigma$ Boo an average metal deficiency of a factor of 4 . From Wallerstein's (1962) correlation between $\delta(U-B)$ and Fe-abundance deficiency, we conclude that the remaining stars on our lists have abundance deficiencies varying from a factor of 2 to 10. Assuming the same initial helium content, it is reasonable to compare

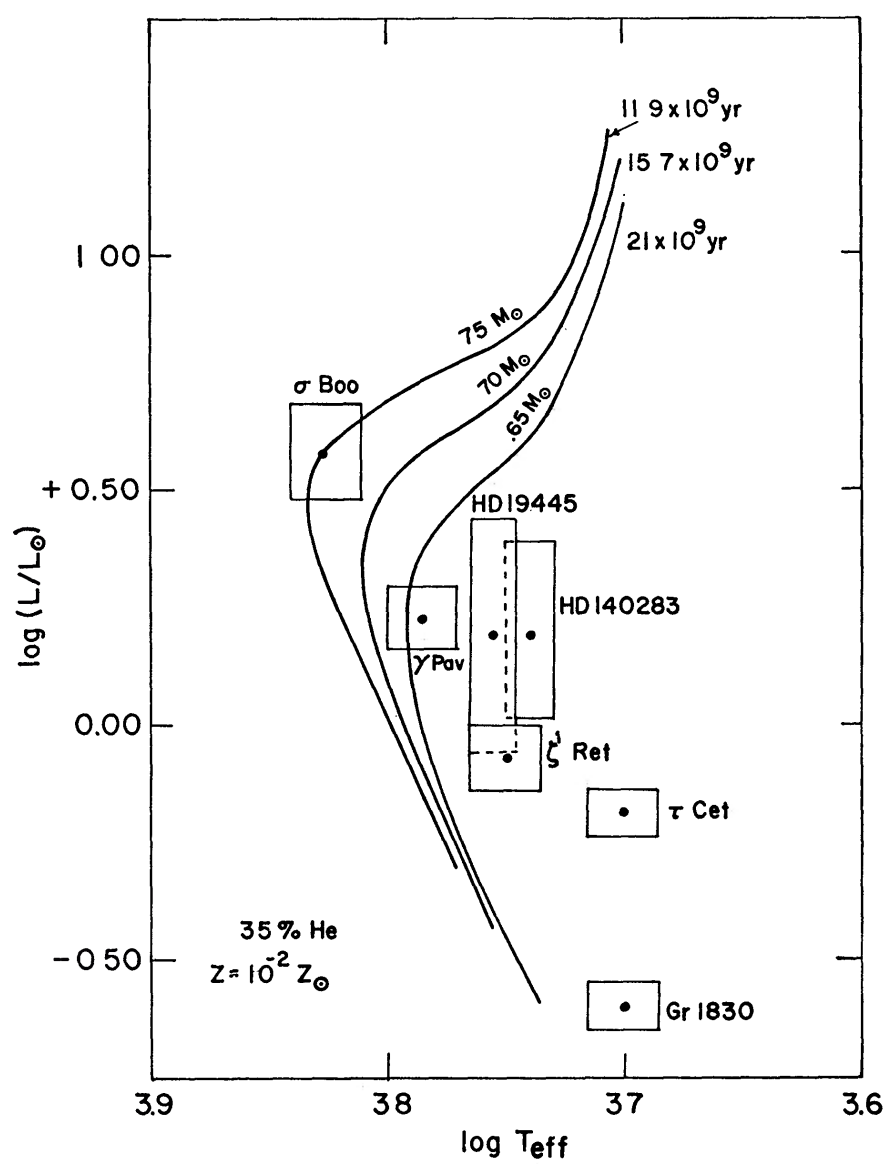

FIG. 11.-Comparison between the deduced positions of 7 subdwarfs on the H-R diagram and the evolutionary tracks of Faulkner and Iben (1966) for high-helium-content Population II stars.

the stars of low metal content with tracks having the same $Z$, since to a first approximation we can neglect the contribution of the metals to the total opacity for low $Z$. Faulkner and Iben (1966) and Iben (1966) have computed a set of evolutionary tracks having the following characteristics:

$$
\begin{gathered}
M / M \odot=0.75,0.70,0.65 \quad \text { for } \quad X=0.65, Y=0.35, Z=2 \times 10^{-4}, \\
M / M \odot=1.20,1.10,1.00 \quad \text { for } \quad X=0.90, Y=0.10, Z=2 \times 10^{-4}, \\
\text { Mixing length/pressure scale height }=0.5 .
\end{gathered}
$$

We reproduce in Figures 11 and 12 Faulkner and Iben's evolutionary tracks, and have superposed on these tracks the positions of the subdwarfs. Note that the ages shown in 
these figures represent the total time required to reach the indicated positions on the giant branch. The time elapsed between the main-sequence turnoff and the indicated position on the giant branch represents less than 10 per cent of the evolutionary history. I [From Figure 11, in which the high-helium-abundance tracks are plotted, we note that the ages deduced for HD 140283, HD 19445, $\tau$ Ceti, $\gamma$ Pav, and $\zeta^{1}$ Ret exceed $20 \times 10^{9}$ years. A uniform decrease of a minimum of 0.04 in the log of the $T_{\text {eff }}$ predicted from the Iben-Faulkner stellar models is required in order to reconcile most of the subdwarf ages with the age of the Universe deduced from the Hubble constant, namely, 8 to $13 \times 10^{9}$

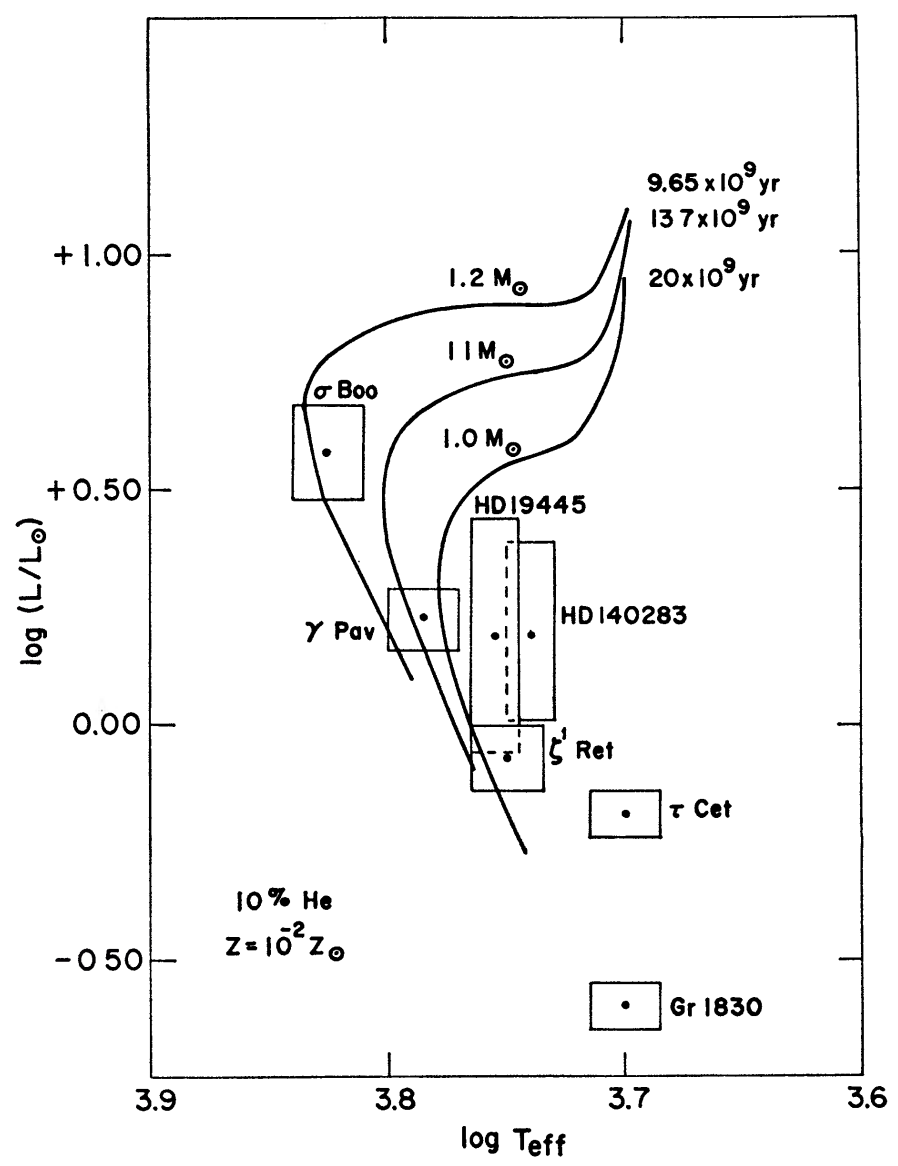

FIG. 12.-Comparison between the deduced positions of 7 subdwarfs on the H-R diagram and the evolutionary tracks of Faulkner and Iben (1966) for low-helium-content Population II stars.

years. Even with this correction, $\tau$ Ceti, HD 140283, and HD 19445 would have ages in excess of $15 \times 10^{9}$ years. In addition, we must also recall that any errors in $T_{\text {eff }}$ are most likely to shift the positions of these stars even further to the right. A shift in the evolutionary tracks of 0.04 in $\log T_{\text {eff }}$ is conceivable if we reduce the efficiency of convection. Such a decrease is qualitatively consistent with the extremely low turbulent velocities observed for subdwarfs. Shifts much in excess of 0.04 in $\log T_{\text {eff }}$ would force $\sigma$ Boo, $\gamma$ Pav, and $\zeta^{1}$ Ret well to the left of the predicted main sequence.

In Figure 12, in which are plotted the low-helium-abundance tracks, we find the main sequence well defined by Gr 1830, $\zeta^{1}$ Ret, $\gamma$ Pav, and $\sigma$ Boo, while $\tau$ Ceti, HD 140283, and HD 19445 lie somewhat to the right. A shift of about 0.04 in $\log T_{\text {eff }}$ in the computed tracks brings all stellar ages into reasonable agreement with the Hubble constant age. 
Thus the low-helium-abundance tracks are to be preferred on this basis. However, as Faulkner and Iben (1966) point out, the high-helium-abundance models provide a better qualitative match with the globular cluster H-R diagrams than do the low Y models. A more definitive statement regarding the helium content of Population II stars must probably await the analysis of horizontal-branch stars in which a helium abundance can be determined spectroscopically.

As a concluding remark concerning the subdwarf ages, we can only state that in several cases the ages may be uncomfortably larger than $10 \times 10^{9}$ years.

In Paper II, we will describe the results of an abundance analysis for the extreme subdwarfs HD 140283 and HD 19445. Subsequent papers in this series will be concerned with analysis of $\zeta^{1}$ Ret, $\gamma$ Pav, and $\sigma$ Boo.

We wish to thank Prof. Icko Iben for many valuable comments made in the course of several discussions and for supplying in advance of publication his and Faulkner's Population II stellar models.

\section{REFERENCES}

Aller, L. H., and Greenstein, J. L. 1960, Ap. J. Suppl., 5, 139.

Burbidge, E. M., and Burbidge, G. R. 1956, Ap. J., 124, 116.

Chamberlain, J. W., and Aller, L. H. 1951, Ap. J., 114, 52.

Danziger, I. J. 1966b, Ap. J., 143, 527.

- $1966 a$, private communication.

Eggen, O. J. 1955, A.J., 60, 65.

- - 1962, R.O.B., 51, E79.

Eggen, O. J., and Sandage, A. R. 1962, Ap. J., 136, 735.

Faulkner, J., and Iben, I. 1966, Ap. J., 144, 995.

Gingerich, O. J., and Rich, J. 1967, in preparation.

Goldberg, L., Müller, E. A, and Aller, L. H. 1960, Ap. J. Suppl., 5, 1.

Iben, I. 1966, private communication.

Kalkofen, W., and Strom, S. E. 1966, J. Quant. Spectrosc. and Rad. Transfer, 6, 653.

Melbourne, W. G. 1960, Ap J., 132, 101.

Oke, J. B. 1965, Ann. Rev. Astr. and Ap., 3, 26.

Sandage, A. R., and Eggen, O. J. 1959, M.N., 119, 278.

Schwarzschild, M., and Schwarzschild, B. 1950, Ap. J., 112, 248.

Schwarzschild, M., Searle, L., and Howard, R. 1955, Ap.J., 122, 353.

Searle, L., and Oke, J. B. 1962, Ap. J., 135, 790.

Strom, S. E., and Avrett, E. H. 1965, Ap. J. Suppl., 12, 1.

Strom, S. E, and Cohen, J. G. 1966, submitted to A P. J. (Paper II).

Strom, S. E., and Kurucz, R. 1966, J. Quant. Spectrosc. and Rad. Transfer, 6, 591.

Swihart, T., and Fischel, D. 1961, Ap. J. Suppl., 5, 291.

Wallerstein, G. 1962, Ap.J. Suppl., 6, 407.

Willstrop, R. V. 1965, Mem. R.A.S., 69, 83. 